\title{
PROGNOSIS IN LONG-TERM IMMUNOSUPPRESSIVE TREATMENT OF REFRACTORY CHRONIC INFLAMMATORY DEMYELINATING POLYRADICULONEUROPATHY
}

\author{
Mark B. Bromberg, ${ }^{*}$ Eva L. Feldman, Safwan Jaradeh and James W. Albers \\ Department of Neurology, University of Michigan Medical Center, Ann Arbor, MI 48109, U.S.A.
}

(Received 4 July 1991)

\begin{abstract}
Treatment of chronic inflammatory demyelinating polyneuropathy (CIDP) frequently includes use of immunosuppressive agents. Controlled treatment trials demonstrating efficacy are available only for prednisone and therapeutic plasma exchange (TPE). When these fail to achieve lasting clinical improvement after reduction or cessation of therapy, subsequent regimens are empiric, often leading to prolonged immunosuppression. It is not possible to predict who will respond to which agent and when. Administered individually, immunosuppressive agents may pose an acceptable risk, but cumulative effects of multiple agents in refractory patients may suppress the immune system and contribute to increased morbidity and mortality. Treatment difficulties with refractory CIDP patients have not been emphasized, and long-term effects of immunosuppression have focused on the risk of malignancy. In reviewing our clinical experience treating over $100 \mathrm{CIDP}$ patients we identified approximately 20 patients who could be considered refractory to multiple immunosuppressive therapies and dependent upon long-term intermittent TPE. Two of these patients exemplify the morbidity associated with CIDP and its associated treatment. Our review of the clinical course of these patients raised issues about the use of multiple immunosuppressive agents, long-term goals, and long-term prognosis in CIDP.
\end{abstract}

Chronic inflammatory polyradiculoneuropathy CIDP Treatment of polyneuropathy Long-term immunosuppression

\section{INTRODUCTION}

Chronic inflammatory demyelinating polyneuropathy (CIDP) may follow a progressive or relapsing clinical course [1]. Treatment of CIDP is based on altering the immune system $[1,2]$. The experience of our Neuromuscular Clinic in treating over $100 \mathrm{CIDP}$ patients during the past decade is similar to that reported from other neuromuscular centers in that (1) the majority of patients respond satisfactorily to

*All correspondence should be addressed to: Dr M. B. Bromberg, Department of Neurology, University of Michigan Medical Center, Taubman Center 1920/0316, 1500 East Medical Center Drive, Ann Arbor, MI 481090316, U.S.A. [Tel: (313) 936-7165]. a single agent or combination treatment regimen of therapeutic plasma exchange (TPE) and corticosteroids; (2) a substantial percentage of patients respond less well to corticosteroids and experience relapses which require treatment with immunosuppressive agents; and (3) a small group of patients are refractory to multiple agents and become bed or wheelchair bound, and may die from the disease $[1,3,4]$. For the latter two groups of CIDP patients, the choice of subsequent treatment regimens become problematic $[1,2]$. There are few controlled trials of single [5-7] and multiple agents [8], and the choice of particular agents is guided mainly by case reports. 
In reviews of therapeutic response in CIDP patients, treatment failures and circumstances surrounding patient morbidity and mortality (other than from respiratory failure) are rarely reported $[1,3,4]$. From our clinical experience in treating refractory CIDP patients, two patients stand out because of similar courses. Both were dependent upon TPE despite receiving multiple immunosuppressive therapies given in an attempt to prolong remission and reduce disability and both had similar medical complications occurring late in their treatment. The clinical experience with these two patients raises issues about use of multiple immunosuppressive agents, long-term goals, and long-term prognosis in severe CIDP.

\section{CASE PRESENTATIONS, TREATMENT}

\section{RESPONSES, AND POST-MORTEM FINDINGS}

Both patients were men in good health and with no pertinent past medical, family and toxic exposure histories, except patient $\mathbf{A}$ had mild non-insulin dependent diabetes mellitus diagnosed and treated with chlorpropamide 6 months after onset of neurologic symptoms. Medical examinations, laboratory blood studies, skeletal X-ray surveys, and bone marrow biopsies were normal. Neither had anti-myelin associated antibody activity. Motor nerve conduction studies fulfilled criteria suggestive of acquired primary demyelination [9]. Needle electromyography revealed chronic denervation. Late in the illness, patient B had only 1 or 2 voluntary motor units in intrinsic hand muscles.
TPEs were performed on continuous flow machines. Remission was induced with a course of 5 TPE treatments performed every other day, and remission was maintained by single exchanges at intervals from twice weekly to once every 3 weeks.

Patient $A$ presented at age 58 with distal numbness and weakness and over 6 months became bedridden (MRC grade 3+; functional scale grade 4, see Fig. 1). An IgG kappa gammopathy was found. His initial response to combined oral prednisone and a course of TPE (schedule and dosages, see Table 1 and Fig. 1) was rapid, with improved limb strength within days, ambulation with a cane by 2 months, and bicycle riding by 5 months. Exacerbations which affected ambulation often occurred precipitously over 1-3 days, but response was prompt and reliable following 5 TPEs. Sustained improvement without TPE was not accomplished and he remained dependent upon periodic weekly or twice weekly TPE despite receiving cyclophosphamide, TLI, and melphalan. Increasing the interval between TPE treatments resulted in deterioration on each occasion it was attempted.

At final hospitalization for unexplained and worsening jaundice, patient A was ambulatory, but dependent upon weekly TPEs. Hepatitis A and $B$ markers were normal, and there was no evidence of biliary obstruction. He developed thrombocytopenia and a coagulopathy which necessitated a reduction in TPE frequency. $\mathrm{He}$ became weaker, developed mild adult respiratory distress syndrome, and temporarily required

Table 1. Summary of immunosuppressive therapy (see also Fig. 1)

\begin{tabular}{lll}
\hline Treatment & \multicolumn{1}{c}{ Patient A } & \multicolumn{1}{c}{ Patient B } \\
\hline Therapeutic plasma exchange & 215 & 111 \\
(No.) & $3 / 83-8 / 87$ & $6 / 83-11 / 87$ \\
Prednisone & $40 \mathrm{mg}$ QOD-100 mg QD & $10 \mathrm{mg}$ QOD-60 mg QD \\
(PO; min-max) & $3 / 83-8 / 87$ & $4 / 83-12 / 87$ \\
Methylprednisolone & $2 \mathrm{~g} \times 2$ doses & $1 \mathrm{~g} \times 3$ doses \\
(IV) & $1 / 87$ & $3 / 87$ \\
Azathioprine & $150 \mathrm{mg} \mathrm{QD}$ & $150-200 \mathrm{mg}$ QD \\
(PO; min-max) & $1 / 84$ & $9 / 83-2 / 84$ \\
& $($ systemic reaction) & (leukopenia, count 2000) \\
Cyclophosphamide & $100-200 \mathrm{mg}$ QD & \\
(PO; min-max) & $6 / 84-11 / 84$ & \\
& (leukopenia, count 2000) & \\
Melphalan & $12 \mathrm{mg}$ QD $\times 4$ doses & $11 \mathrm{mg}$ QD $\times 4$ doses \\
(PO) & $3 / 87,4 / 87,5 / 87$ & $6 / 87,7 / 87,8 / 87$ \\
Total lymphoid irradiation & Mantle: 1950 rad & Mantle: 2160 rad \\
& lnverted Y: 1950 rad & Inverted Y: 120 rad \\
& $6 / 85-8 / 85$ & (lis6-12/86 \\
& & (leukopenia, count 2000) \\
\hline
\end{tabular}



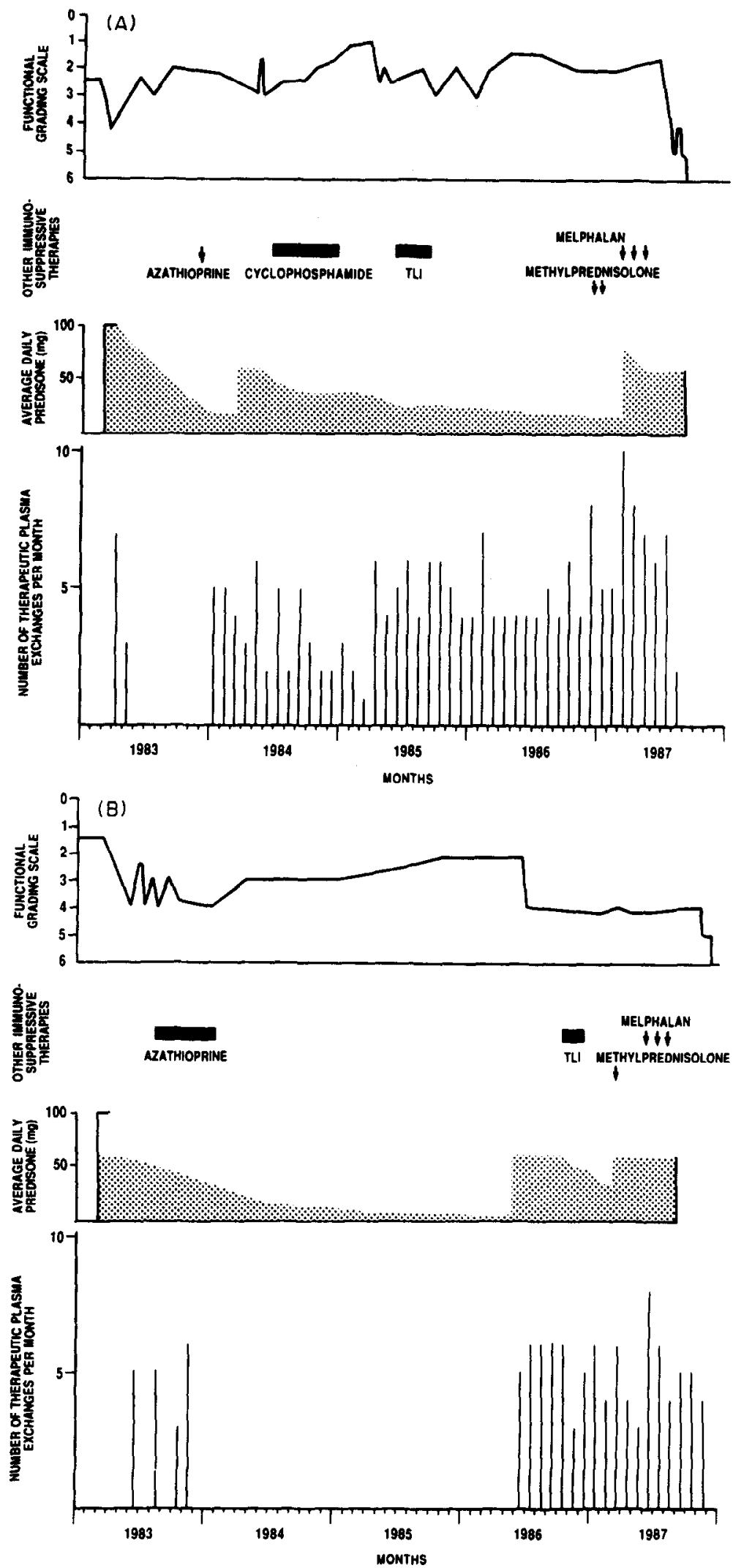

Fig. 1. Clinical course and treatment schedules. Upper trace: functional grading scale of strength*. Second trace: time and duration of immunosuppressive drugs (see Table for dosages). Third trace: averaged daily prednisone dosage. Lower trace: number of TPE treatments per month. (A) Patient A and (B) patient B. Functional grading scale: 0-healthy; 1-minor signs or symptoms; 2 -able to walk $5 \mathrm{~m}$ without a walker or equivalent support; 3-able to walk $5 \mathrm{~m}$ with a walker or support; 4-bed or chairbound (unable to walk $5 \mathrm{~m}$ with a walker or support); 5 -requires assisted ventilation; 6 -dead. 
ventilatory support. Pneumonia developed and he died at age $63,5 \frac{1}{2}$ years after onset of neurologic symptoms. Post-mortem examination revealed disseminated aspergillosis and candidemia, with cytomegalic virus inclusions in lung and liver. The hepatobiliary system was unremarkable. The liver had confluent central zone necrosis without evidence of lymphocytic infiltration or fibrosis or alterations of the portal system. No amyloid was detected.

Patient $B$ presented at age 55 with acral numbness and weakness which progressed over $2 \frac{1}{2}$ years (MRC grade 4; functional grade 2, see Fig. 1). An IgM lambda gammopathy was discovered. There was no response to 3 months of oral prednisone (schedule and dosages, see Table 1 and Fig. 1), but he responded promptly to a course of 5 TPEs. This was short lived and his condition deteriorated until he was bedbound. He slowly improved over many months on combined prednisone and azathioprine therapy to the point of cautious unaided walking. An abrupt relapse associated with a viral-like illness caused him to become wheelchair-bound. He never improved despite use of azathioprine, TLI, and melphalan. Maintenance of limited strength remained dependent upon frequent TPEs.

His final admission was for bloody diarrhea. Adult respiratory distress syndrome developed and ventilator support was required. He developed coagulase negative staphylococcus bacteremia and candidemia. He became hypotensive and comatose, and died at age 63, $7 \frac{1}{2}$ years after onset of neurologic symptoms. Postmortem findings included mucosal infarcts of the large bowel, bacterial pneumonitis, and cytomegalic viral inclusions in the lungs. No adenopathy, marrow infiltrates, or amyloid was found.

\section{DISCUSSION}

The relapsing or progressive forms of CIDP frequently require protracted treatment. Therapeutic modalities demonstrated to be effective in controlled trials include TPE [6] and prednisone [5]. Sustained remission with TPE alone is rare and concurrent use of corticosteroids is usually required [6]. Prolonged treatment with prednisone is associated with troublesome side effects $[1,10,11]$. For some patients, high dose prednisone may not be effective [1-4], and under these circumstances immunosuppressive agents are commonly used [2].
The choice of subsequent therapy becomes uncertain, and without controlled studies, the choice is guided only by case reports. Immunosuppressive agents reported effective at time of treatment of our two patients, or subsequently, included azathioprine [12], cyclophosphamide [2], melphalan [13], polyinosinic-polycytidylic acid [14], TLI [15], cyclosporin [16], and i.v. gammaglobulin $[7,17]$.

Our patients were examples of patients with relapsing and refractory CIDP who had severe disability and common side effects of prednisone. For each, azathioprine was chosen [12] but was not tolerated; and patient $A$ had a systemic reaction with the first doses [18], and patient B had leukopenia after 5 months, which subsequently resolved. Cyclophosphamide was tolerated by patient $\mathrm{A}$ but was discontinued because of transient leukopenia. TLI was administered because of continued weakness and a report of 4 patients with refractory CIDP who showed improvement 3 months after treatment with TLI with no major side effects [15]. Radiation therapy was well tolerated with only mild and transient leukopenia in patient $B$, but there were no remissions during follow up periods of 10-24 months. Although there was no evidence for plasma cell malignancy, melphalan was given because of a good response in refractory dysproteinemic polyneuropathy [13]. Neither patient lived long enough to reliably assess drug response. Melphalan has been associated with pulmonary fibrosis [19], but pulmonary difficulties began after melphalan was discontinued, and there was no pathologic evidence for fibrosis or atypical alveolar cells.

Both of our patients had a monoclonal gammopathy of uncertain significance (MGUS). It is not clear if CIDP with MGUS represents a separate disorder or is part of a spectrum of CIDP [20]. The presence of a MGUS in CIDP is common, occurring in up to $30 \%$ of patients [21], and our patients followed a clinical course typical for CIDP before and early-on after treatment was initiated. Without evidence for an underlying tumor at postmortem examination we feel the MGUS did not make the clinical course of our two patients unique.

As the therapeutic regimen is extended, prolonged immunosuppression becomes a concern. Most reviews of complications of long-term immunosuppressive therapy focus on increased risk of malignancy $[22,23]$. There was no evidence of tumor in our patients. However, 
both patients at post-mortem examination had disseminated cytomegalic inclusion bodies and evidence of multiple fungal and bacterial infections suggesting marked impairment of immunologic function. Consideration must be given to accumulated effects of multiple immunosuppressive agents to account for marked immunosuppression. Of the agents used, TLI induces long lasting suppression of cell mediated immunity [24]. Long-term side effects of TLI alone [25], or with multiple immunosuppressive therapies, have been discussed [26]. Conditions and circumstances which contribute to morbidity and mortality under these circumstances include bacterial and viral infections, greater degree of physical disability, and patient age greater than 65 years $[25,27]$.

The difficult clinical issue is the CIDP patient who never responds to treatment or who responds temporarily but relapses frequently despite vigorous treatment with multiple immunosuppressive agents. There are no obvious means of early identification of such patients. Continued efforts with more immunosuppressive agents must be tempered by recognition of possible cumulative effects on the immune system. The pressures to treat refractory CIDP patients with vigorous immunosuppression are different than the pressures to treat other patients groups, such as transplant patients. Accordingly, other factors in CIDP patients, such as the extent of clinical deficits (profound sensory loss and weakness), duration of illness, and advanced age must be considered and should influence choice of drugs and duration of trials [6]. In particular, in patients with evidence of substantial axonal loss on electrodiagnostic studies [6], treatment response is difficult to evaluate because it may be delayed by months. In addition, the reinnervation capacity in older individuals may be limited and partially satiated by age-related loss of motor neurons or axons [28, 29]. In such patients, multiple therapeutic agents may be used and abandoned or supplemented with other agents before it would have been possible to judge their effectiveness. Attempts to find agents which will improve patient function and induce permanent remission are reasonable goals. However, recognition early on that patients who possess some of the factors listed above may do better and have fewer side effects with long term, periodic temporizing therapies such as TPE and i.v. immunoglobulin is important $[30,31]$.

\section{REFERENCES}

1. Dyck PJ, Lais AC, Ohta M, Bastron JA, Okazaki H, Groover RV. Chronic inflammatory polyradiculoneuropathy. Mayo Clin Proc 1975; 50: 621-637.

2. Dalaka MC, Engel WK. Chronic relapsing (dysimmune) polyneuropathy: pathogenesis and treatment Ann Neurol 1981; 9(Suppl.1): 134-145.

3. McCombe PA, Pollard JD, McLeod JG. Chronic inflammatory demyelinating polyradiculoneuropathy. Brain 1987; 110: 1617-1630.

4. Barohn RJ, Kissel JT, Warmolts JR, Mendell JR Chronic inflammatory demyelinating polyradiculoneuropathy. Arch Neurol 1989; 46: 878-884.

5. Dyck PJ, O'Brien PC, Oviatt KF, Dinapoli RP, Daube JR, Bartleson JD, Mokri B, Swift T, Low PA, Windebank AJ. Prednisone improves chronic inflammatory demyelinating polyradiculoneuropathy more than no treatment. Ann Neurol 1982; 11: 136-141.

6. Dyck PJ, Daube J, O'Brien P, Pineda P, Low PA, Windebank AJ, Swanson C. Plasma exchange in chronic inflammatory demyelinating polyradiculoneuropathy. N Engl J Med 1986; 314: 461-465.

7. Van Doorn PA, Brand A, Strengers PFW, Meulstee J, Vermeulen M. High-dose intravenous immunoglobulin treatment in chronic inflammatory demyelinating polyneuropathy. Neurol 1990; 40: 209-212.

8. Dyck PJ, O'Brien P, Swanson C, Low P, Daube J. Combined azathioprine and prednisone in chronic inflammatory-demyelinating polyneuropathy. Neurol 1985; 35: 1173-1176.

9. Albers JW, Kelly JJ. Acquired inflammatory demyelinating polyneuropathies: clinical and electrodiagnostic features. Muscle Nerve 1989; 12: 435-451.

10. Sghirlanzoni A, Peluchetti D, Mantegazza R, Fiacchino F, Cornelio F. Myasthenia gravis: prolonged treatment with steroids. Neurol 1984; 34: 170-174.

11. Pascuzzi RM, Coslett HB, Johns TR. Long-term corticosteroid treatment of myasthenia gravis: report of 116 patients. Ann Neurol 1984; 15: 291-298.

12. Pentland B, Adams GGW, Mawdsley C. Chronic idiopathic polyneuropathy treated with azathioprine. J Neurol Neurosurg Psychiat 1982; 45: 866-869.

13. Donofrio PD, Albers JW, Greenberg HS, Mitchell BS Peripheral neuropathy in osteosclerotic myeloma: clinical and electrodiagnostic improvement with chemotherapy. Muscle Nerve 1984; 7: 137-141.

14. Engel WK, Cuneo RA, Levy HB. Polyinosinicpolycytidylic acid treatment of neuropathy. Lancet 1978; 1: 503-504.

15. Rosenberg NL, Lacy JR, Kennaugh RC, Holers VM, Neville HS, Kotzin BL. Treatment of refractory chronic demyelinating polyneuropathy with lymphoid irradiation. Muscle Nerve 1985; 8: 223-232.

16. Hodgkinson SJ, Pollard JD, McLeod JG. Cyclosporin $A$ in the treatment of chronic demyelinating polyradiculoneuropathy. J Neurol Neurosurg Psychiat 1990; 53: 327-330.

17. Van Doorn PA, Vermeulen M, Brand A, Mulder PFH, Busch HFM. Intravenous immunoglobulin treatment in patients with chronic inflammatory demyelinating polyneuropathy. Arch Neurol 1991; 48: 217-220.

18. Kissel JT, Levy RJ, Mendell JR, Griggs RC. Azathioprine toxicity in neuromuscular disease. Neurol 1986; 36: 35-39.

19. Westerfield BT, Michalski JP, McCombs C et al. Reversible melphalan-induced lung damage. Am J Med 1980; 68: 767-771.

20. Dyck PJ. Intravenous immunoglobulin in chronic inflammatory demyelinating polyradiculoneuropathy and in neuropathy associated with IgM monoclonal gammopathy of unknown significance. Neurol 1990; 40: $327-328$. 
21. Bromberg MB. Comparison of electrodiagnostic criteria for primary demyelination in chronic polyneuropathy. Muscle Nerve 1991; 14: 968-976.

22. Kinlen LJ, Sheil AGR, Peto J, Doll R. Collaborative United Kingdom-Australasian study of cancer in patients treated with immunosuppressive drugs. Br Med J 1979; 2: 1461-1466.

23. Boitard C, Bach J-F. Long-term complications of conventional immunosuppressive treatment. Adv Nephrol 1898; 18: 335-354.

24. Kotzin BL, Strober S. Total lymphoid irradiation. Clin Immunol Allergy 1984; 4: 331-358.

25. Zvaifler NJ. Fractionated total lymphoid irradiation: a promising new treatment for theumatoid arthritis? yes, no, maybe. Arthritis Rheum 1987; 30: 109-114.

26. Wilder RL. Tretment of the patient with rheumatoid arthritis refractory to standard therapy. JAMA 1988; 259: 2446-2449.
27. Myers LW, Ellison GW, Fahey JL, Tesler A, Gottlieb MS. Clinical drawbacks of total lymphoid irradiation: the cons. Neurol 1988; 38(Suppl. 2): 38-40.

28. Stålberg E, Thiele B. Motor unit fiber density in the extensor digitorum communis muscle. J Neurol Neurosurg Psychiat 1975; 38: 874-880.

29. Stålberg E. Electrophsiological studies of reinnervation in ALS. In: Rowland LR, Ed. Human Motor Neuron Diseases, New York: Raven Press; 1982: 47-57. 30. Beydoun SR, Engel WK, Karofsky, Schwartz MU. Long-term plasmapheresis therapy is effective and safe in children with chronic relapsing dysimmune polynesuyropathy. Rev Neurol (Paris) 1990; 146: 2, 123-127.

31. Faed JM, Day B, Pollock M, Taylor PK, Nukada H, Hammond-Tooke GD. High-dose intravneous human immunoglobulin in chronic inflammatory demyelinating polyneuropathy. Neurol 1989; 39: 422-425. 